\title{
Selective deletion of leptin receptor in neurons leads to obesity
}

\author{
Paul Cohen, ${ }^{1}$ Connie Zhao,${ }^{1}$ Xiaoli Cai, ${ }^{1}$ Jason M. Montez, ${ }^{1}$ S. Christy Rohani, ${ }^{1}$ \\ Paul Feinstein, ${ }^{2}$ Peter Mombaerts, ${ }^{2}$ and Jeffrey M. Friedman ${ }^{1,3}$ \\ ${ }^{1}$ Laboratory of Molecular Genetics, \\ ${ }^{2}$ Laboratory of Developmental Biology and Neurogenetics, and \\ ${ }^{3}$ Howard Hughes Medical Institute, The Rockefeller University, New York, New York, USA \\ Address correspondence to: Jeffrey M. Friedman, Howard Hughes Medical Institute, \\ The Rockefeller University, 1230 York Avenue, Box 305, New York, New York 10021, USA. \\ Phone: (212) 327-8800; Fax: (212) 327-7420; E-mail: friedj@mail.rockefeller.edu. \\ Paul Cohen and Connie Zhao contributed equally to this work. \\ Received for publication August 6, 2001, and accepted in revised form September 10, 2001.
}

Animals with mutations in the leptin receptor (ObR) exhibit an obese phenotype that is indistinguishable from that of leptin deficient $o b / o b$ mice. ObR is expressed in many tissues, including brain, and the relative importance of leptin's effects on central versus peripheral sites has not been resolved. To address this, we generated mice with neuron-specific $\left(\mathrm{ObR}^{\mathrm{Syn}} \mathrm{KO}\right)$ and hepatocyte-specific $\left(\mathrm{ObR}^{\mathrm{Alb}} \mathrm{KO}\right)$ disruption of ObR. Among the $\mathrm{ObR}^{\mathrm{Syn}} \mathrm{KO}$ mice, the extent of obesity was negatively correlated with the level of ObR in hypothalamus and those animals with the lowest levels of ObR exhibited an obese phenotype. The obese mice with low levels of hypothalamic ObR also show elevated plasma levels of leptin, glucose, insulin, and corticosterone. The hypothalamic levels of agouti-related protein and neuropeptide Y RNA are increased in these mice. These data indicate that leptin has direct effects on neurons and that a significant proportion, or perhaps the majority, of its weight-reducing effects are the result of its actions on brain. To explore possible direct effects of leptin on a peripheral tissue, we also characterized $\mathrm{ObR}^{\mathrm{Alb}} \mathrm{KO}$ mice. These mice weigh the same as controls and have no alterations in body composition. Moreover, while $d b / d b$ mice and $\mathrm{ObR}^{\mathrm{Syn} I} \mathrm{KO}$ mice have enlarged fatty livers, $\mathrm{ObR}^{\mathrm{Alb}} \mathrm{KO}$ mice do not. In summary, these data suggest that the brain is a direct target for the weight-reducing and neuroendocrine effects of leptin and that the liver abnormalities of $d b / d b$ mice are secondary to defective leptin signaling in the brain.

This article was published online in advance of the print edition.

The date of publication is available from the JCI website, http://www.jci.org.

J. Clin. Invest. 108:1113-1121 (2001). DOI:10.1172/JCI200113914.

\section{Introduction}

Leptin is an adipocyte hormone that functions as an afferent signal in a negative feedback loop that regulates energy balance (1-3). Mice with mutations in leptin $(o b / o b)$ or its receptor $(d b / d b)$ are hyperphagic and severely obese (4-6). These mutant mice also manifest a large number of metabolic and endocrine abnormalities including diabetes, hypercortisolemia, infertility, and cold intolerance $(7,8)$. These $o b / o b$ and $d b / d b$ mice also have enlarged, steatotic livers $(9,10)$. The fatty liver is in part a result of an increased rate of hepatic lipogenesis, which is also thought to contribute to the development of obesity (11).
Treatment of $o b / o b$ mice with leptin reduces food intake and body weight and corrects the metabolic and endocrine defects associated with leptin deficiency. Leptin treatment also normalizes the hepatomegaly and associated elevations in hepatic glycogen and lipid (12). Infusions of leptin into wild-type mice in physiological amounts results in a dose-dependent reduction in food intake and body weight (13-15). This metabolic response to leptin in both $o b / o b$ and wild-type mice is novel and is not solely a consequence of its anorectic effects (16). Intracerebroventricular (ICV) leptin has similar effects, but at much lower doses, suggesting that leptin has direct effects on brain $(15,17)$.

The leptin receptor, which has five splice variants (ObRa-e), is broadly expressed, however, and the relative importance of leptin's effects on brain versus peripheral sites is unclear $(18,19)$. This distinction is of importance because leptin deficiency is associated with myriad abnormalities in many tissues including liver. Although leptin has been suggested to act directly to activate signal transduction and deplete triglycerides, the contribution of these actions to body weight homeostasis, deposition of lipid in peripheral sites, and neuroendocrine function in vivo is untested $(20,21)$. To determine the role of leptin action in the central nervous system and the periphery, we are systematically deleting ObR in a tissue-specific fashion using the Cre-loxP system. Here we present data from mice with either neuronal or hepatocyte-specific deletions of ObR.

\section{Methods}

ObR floxed mice. A 12-kb genomic clone containing the first coding exon of ObR was isolated. A single loxP element was PCR-amplified from the plasmid pNeoTKLox. The oligonucleotide primers were engineered to introduce a BamHI-NcoI double restriction site at one end and a BamHI site at the other end. After digestion with BamHI, the product was ligated into the BamHI site of the genomic clone, resulting in the clone BNLOXB with a single LoxP site upstream of the first exon. A loxP-NeoHSV-TK-LoxP element was isolated by digesting pNeoTKLox with BstXI and SalI. The fragment was blunt-ended and cloned into BglII digested blunt-ended 
BNLOXB. This clone, denoted ObR-loxSS1-lox, was double digested with AflII and KpnI, blunt-ended, and re-ligated to generate the targeting vector. The excised 500-bp AflII-KpnI fragment was used as probe 1 . The targeting vector was linearized with SalI and electroporated into 129/SV embryonic stem (ES) cells. Cells were selected with G418, and surviving clones were screened for homologous recombination. Positive clones were transiently transfected with the Cre recombinase expressing plasmid, p0G231 (kindly provided by $\mathrm{S}$. O'Gorman) (22). Cells were then subjected to ganciclovir selection, and surviving clones were checked by Southern blotting using probe 2 , which is within exon 2, to confirm the deletion of the Neo-HSV-TK cassette (type II deletion). ES cells with the correct genotype $\left(O b R^{f l o x /+}\right)$ were injected into C57BL/6 blastocysts, and resulting chimeras were bred with C57BL/6 mice to obtain germline transmission. ObRflox/+ mice were crossed to generate the line of $O b R$ floxed mice, denoted $O b R^{f l o x} / f l o x$.

ObR-null mice. ObRflox/+ mice were crossed with transgenic adenovirus EIIA Cre mice (kindly provided by $\mathrm{H}$. Westphal) (23). Given that Cre is expressed early in embryogenesis in this line, progeny were screened by Southern blotting with probe 2 for germline Cre-mediated deletion of the first coding exon (type I deletion). These mice, with the genotype $O b R^{4 /+}$, were crossed to produce homozygous $O b R^{\Delta \Delta}$ mice, designated ObR-null mice.

Conditional deletion of ObR. Neuronspecific deletion of ObR was achieved using synapsinI-Cre transgenic mice (SynI-Cre $(+)$ ) (kindly provided by J. Marth) $(24,25)$. Hepatocyte-specific deletion was achieved using Albumincre transgenic mice (Alb-Cre $(+)$ ). The Alb-Cre transgene (see Figure 5a) was constructed using plasmid NB, which contains $2 \mathrm{~kb}$ of the Albumin promoter enhancer (kindly provided by R. Palmiter) (26). A 2-kb fragment containing the Cre-recombinase gene and a nuclear localization and polyadenylation signal was excised from a separate plasmid by digesting with BglII and blunt-ending and then digesting with KpnI. This fragment was cloned into KpnI-EcoRV digested plasmid NB downstream of the albumin promoter enhancer. The resulting plasmid was digested with NotI and KpnI to release the 4-kb transgene, which was gel purified and injected into fertilized eggs from C57/BL6 $\times$ CBA (F1) mice to produce transgenic mice. The presence of the transgene in both $($ SynI-Cre $(+))$ and (Alb-Cre $(+)$ ) mice was verified by PCR and Southern blotting. Tissue-specific knockout mice were generated by two successive crosses (see Figure 2a). First, $O b R^{\Delta /+}$ mice were crossed with either synapsinI-Cre transgenic mice (SynICre $(+))$ or albumin-cre transgenic mice (Alb-Cre $(+))$ to generate $\mathrm{ObR}^{\Delta /+}$, SynI$\mathrm{Cre}(+)$ mice or $\mathrm{ObR}^{\Delta /+}, \mathrm{Alb}-\mathrm{Cre}(+)$ mice. These mice were then mated to

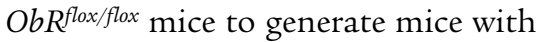
the genotype $\mathrm{ObR}^{\Delta / \text { flox }}$, SynI-Cre $(+)$ or $\mathrm{ObR}^{\Delta / \text { flox }}$, Alb-Cre (+), hereafter designated $\mathrm{ObR}^{\text {SynI }} \mathrm{KO}$ and $\mathrm{ObR}^{\mathrm{Alb}} \mathrm{KO}$, respectively. Mice with the following genotypes were also generated: (a) $\mathrm{ObR}^{\Delta / \text { flox }}$, SynI-Cre(-) and $\mathrm{ObR}^{\Delta / \text { flox }}$, Alb-Cre(-) (heterozygotes); (b) ObRflox $/+$, SynI-Cre $(+)$ and $\mathrm{ObR}^{\mathrm{flox} /+}$, Alb$\mathrm{Cre}(+)$ (referred to as wild-type); and (c) $\mathrm{ObR}^{\mathrm{flox} /+}$, SynI-Cre(-) and $\mathrm{ObR}^{\mathrm{flox} /+}$, Alb-Cre(-) (referred to as wild-type). All animals were housed under controlled temperature $\left(23^{\circ} \mathrm{C}\right)$ and lighting $(12$ hours of light; 12 hours of dark) with free access to food and water. All procedures were in accordance with the guidelines of the Rockefeller University Laboratory Animal Research Center.

Assay of Cre specificity. Genomic DNA was prepared from multiple tissues from ObR ${ }^{\text {flox } /+}$ SynI-Cre $(+)$ and $\mathrm{ObR}^{\mathrm{flox} /+}$ Alb-Cre $(+)$ mice, and 200 ng was PCRamplified using primers flanking the first coding exon of ObR. A schematic of the primer locations is shown in Figure 2b. In tissues expressing Cre recombinase, exon 1 is excised and a single loxP site remains, generating an $\mathrm{ObR}^{\Delta}$ allele. Although primers 1 and 3 can amplify a product from the $\mathrm{ObR}^{\Delta}$ allele, the primers are separated by too great a distance for amplification to occur in the $\mathrm{ObR}^{\text {flox }}$ or $\mathrm{ObR}^{+}$(wild-type) alleles. As a control, primers 1 and 2 were used to amplify both the $\mathrm{ObR}^{\text {flox }}$ and $\mathrm{ObR}^{+}$alleles from all tissues. The $\mathrm{ObR}^{+}$allele produces a slightly smaller product owing to the absence of loxP sequence.

Assay of Cre efficiency. To determine the degree of Cre-mediated recombination, ObR expression levels from all

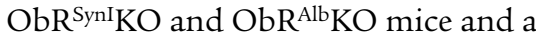
sample of mice with all other possible genotypes were quantitated using Taqman real-time PCR (Perkin Elmer Applied Biosystems, Foster City, California, USA). Total RNA was isolated

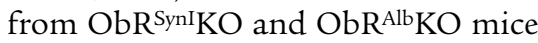
and reverse transcribed into cDNA using random hexamers with the Reverse Transcription Reagents from Roche Molecular Systems (Branchburg, New Jersey, USA). Expression levels were determined using $25 \mathrm{ng}$ of each cDNA sample assayed in duplicate and amplified with the ABI Prism 7700 Sequence Detection System (Perkin Elmer Applied Biosystems). The location of primers and fluorescent probes is indicated in Figure 3b. As amplification occurs, the probe is cleaved, resulting in a signal from a reporter dye that is directly related to the amount of amplicon. Primers were derived from sequences in the $5^{\prime}$ untranslated region (fwd) and the second coding exon (rev). The fluorescent probe, labeled with the FAM dye, contains sequence located within the first coding exon. When this exon is deleted, the probe cannot be cleaved, and no signal is generated. As a control for input amount, each cDNA sample was also amplified using primers and a probe, labeled with the VIC dye, for cyclophilin. Data were analyzed with the ABI Sequence Detector software (Perkin Elmer Applied Biosystems). Every set of reactions contained a set of four serial twofold dilutions of the same liver cDNA source, which was used to generate a standard curve for both ObR and cyclophilin. Amounts of each transcript were calculated from the standard curve, and levels of ObR were corrected for levels of cyclophilin (ObR level/cyclophilin level).

Body composition analysis. Body composition was analyzed as described previously (13). Carcasses were weighed and then oven dried in a $90^{\circ} \mathrm{C}$ oven until weight was constant. The total body water was calculated as the difference between the weights before and after drying. The carcass was then homogenized in a blender, and duplicate aliquots were extracted with a Soxhlet apparatus using a 3:1 mixture of chloroform/methanol. The extracted homogenate was dried overnight and weighed to calculate fat mass and lean mass. 


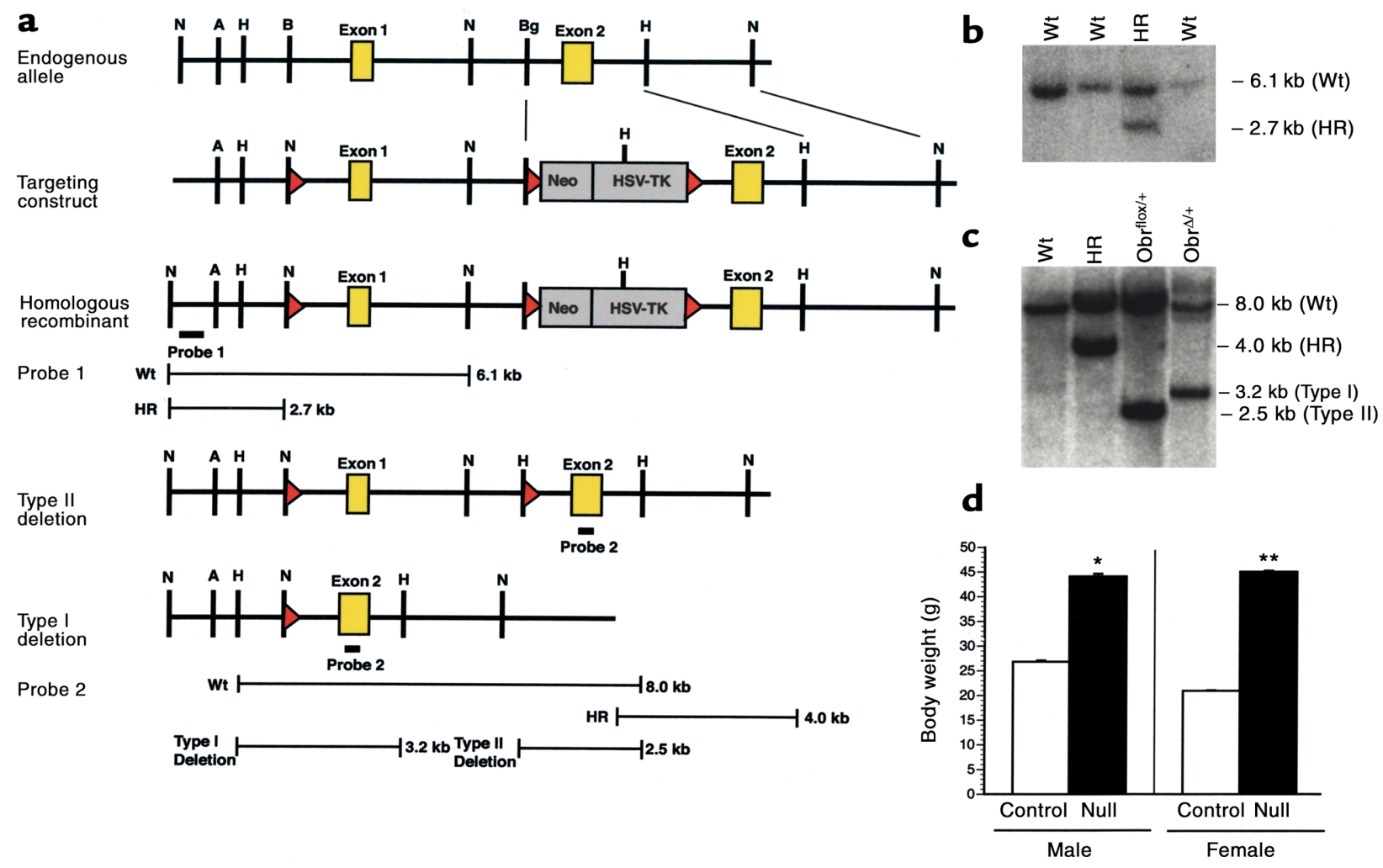

Figure 1

LoxP targeting of the ObR locus. Gene targeting was used to insert loxP sites on either side of the first coding exon of ObR. (a) Restriction maps (from top to bottom) of the genomic locus, targeting vector, homologous recombinant, and the type II and type I deletion alleles. Probe 1 , located outside the targeting construct, was used to screen for homologous recombinants. Probe 2, located in the second coding exon, distinguishes the endogenous allele, homologous recombinants, and type I (deletion of the first coding exon) and II (deletion of the targeting cassette leaving loxP sites on either side of the first coding exon) deletions. A, AfllI; B, BamHI; Bg, BgIII; H, HindIII; N, Ncol. (b) Southern blot analysis of Ncol-digested genomic DNA from ES cell clones using probe 1. The endogenous allele (Wt) and homologous recombinants (HR) migrated at the predicted sizes. (c) Southern blot analysis of Hind-III digested genomic DNA using probe 2. The endogenous allele $(\mathrm{Wt})$, homologous recombinant $(\mathrm{HR})$, and type II deletion were detected from ES cell DNA. ObR ${ }^{\text {flox/+ }}$ mice were generated from the type II deletion and crossed to adenovirus ElIA-Cre mice. The type I deletion was detected in tail DNA from progeny derived from this cross.

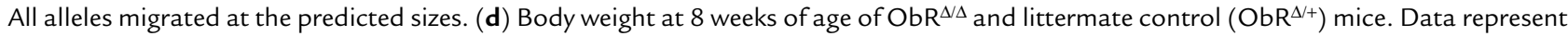
the mean \pm SEM of at least nine animals of each genotype and gender. ${ }^{*} P<0.02 ;{ }^{*} P<0.001$; unpaired Student's $t$ test.

Neuropeptide expression levels. Neuropeptide levels were quantitated by Taqman real-time PCR from individual hypothalami. Expression levels were measured in the obese $\mathrm{ObR}^{\text {SynI }} \mathrm{KO}$ mice, in heterozygote controls, and in female ObR-null mice. Primers and probes were made for agouti-related protein (AGRP), neuropeptide $\mathrm{Y}$ (NPY), melanin concentrating hormone $(\mathrm{MCH})$, proopiomelanocortin (POMC), and cocaine and amphetamine regulated transcript (CART).

Neuroendocrine function. After sacrifice, mice were exsanguinated and blood was collected on EDTA. Plasma was collected after centrifugation and used for all assays. Leptin levels were determined using an ELISA kit from R\&D Systems Inc. (Minneapolis, Minnesota, USA). Insulin levels were determined using an
ELISA kit from Alpco Diagnostics (Windham, New Hampshire, USA). Glucose levels were determined using Trinder reagents from Sigma Chemical Co. (St. Louis, Missouri, USA). Triglyceride levels were determined using an enzymatic kit from Wako Chemical USA Inc. (Richmond, Virginia, USA). Corticosterone, estradiol, testosterone, and thyroxine levels were determined using RIA kits from ICN Radiochemicals Inc. (Costa Mesa, California, USA).

Liver triglyceride quantitation. A total of 40-100 mg of liver from each mouse was homogenized in $4 \mathrm{ml}$ of chloroform-methanol (2:1). A total of $0.8 \mathrm{ml}$ of $50 \mathrm{mM} \mathrm{NaCl}$ was added to each sample. Samples were then centrifuged for 5 minutes at $1,300 \mathrm{~g}$. The lower phase was removed, and duplicate $50-\mu 1$ aliquots were evaporated under $\mathrm{N}_{2}$ gas and then assayed for triglycerides using the Trinder reagent from Sigma Chemical Co. Values are expressed as milligrams of triglyceride per gram of liver.

Statistical analysis. Data are expressed as means \pm SE. Unless otherwise indicated, significance was evaluated using the unpaired Student's $t$ test. Significance of correlation coefficients was evaluated using the $t$ test for correlation.

\section{Results}

We have used the Cre-loxP system to generate mice with tissue-specific deletions of ObR (27). The construction of the targeting plasmid is described above (Figure 1a). The first coding exon of ObR was flanked by loxP sites. This exon contains the signal sequence, and deletion of it by Cre-mediated recombination was predicted to inacti- 
a

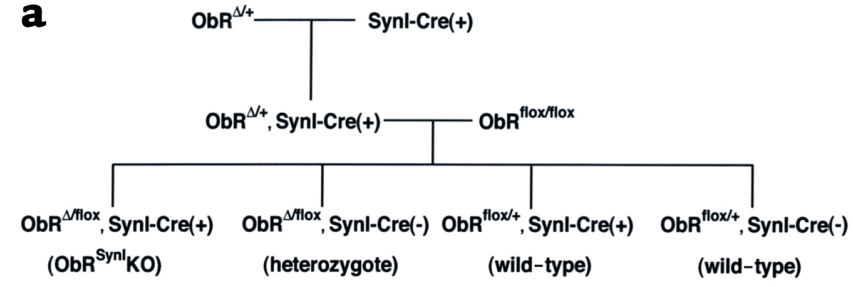

b

Figure 2

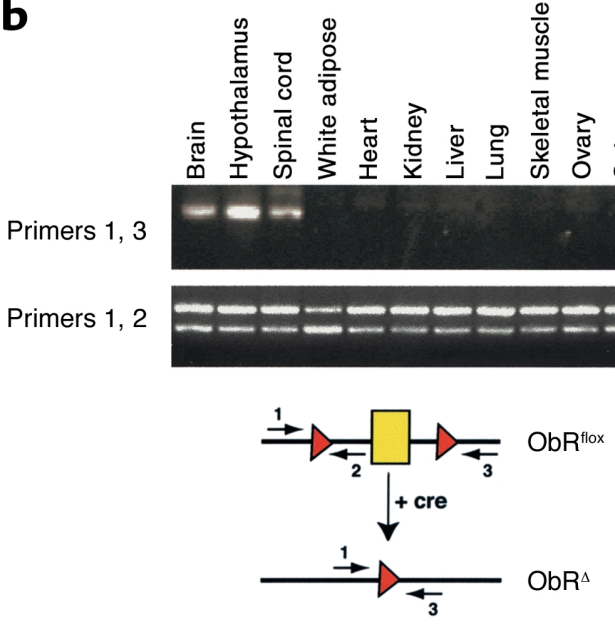

Cre-mediated recombination specifically in the brain of ObR ${ }^{S y n} \mathrm{KO}$ mice. (a) The breeding strategy that was used to generate $\mathrm{ObR}^{\mathrm{Syn}} \mathrm{KO}$ mice and littermate controls. ObR ${ }^{\mathrm{Alb}} \mathrm{KO}$ mice (described below) were generated using the same strategy. (b) Genomic DNA was prepared from several tissues from $\mathrm{ObR}^{\text {flox/+ }}$, Synl-Cre $(+)$ mice and were PCR-amplified using primers flanking the first coding exon of ObR. The locations of the primers are shown on the schematic below the gel. In tissues expressing the Cre recombinase, exon 1 is excised and a single loxP site remains, generating an $\mathrm{ObR}^{\Delta}$ allele. While primers 1 and 3 can amplify a product from the $\mathrm{ObR}^{\Delta}$ allele, in the $\mathrm{ObR}^{\text {flox }}$ or $\mathrm{ObR}^{+}$(wild-type) DNA, the primers are separated by a distance too great for amplification to occur. As a control, primers 1 and 2 were used to amplify both the $\mathrm{ObR}^{\text {flox }}$ allele and the $\mathrm{ObR}^{+}$alleles from all tissues. The $\mathrm{ObR}^{+}$allele produces a slightly smaller product due to the absence of loxP sequences.

vate all splice variants. After transfection into mouse ES cells, three of 72 G418-resistant clones had undergone homologous recombination (Figure 1b). The targeting cassette was deleted by transiently transfecting one of the homologous recombinant ES cell lines with a plasmid expressing Cre recombinase and screening for clones with a type II deletion (Figure 1a). Clones with the correct genotype, $\mathrm{ObR}^{f l o x /+}$, were confirmed by Southern blotting and injected into C57BL/ 6 blastocysts (Figure $1 \mathrm{c})$. Breeding of chimeras confirmed that animals with a germline insertion of loxP sites into ObR had been generated. Mice homozygous for the floxed allele, $O b R^{\text {flox } f \text { flox }}$, were generated by crossing $O b R^{f l o x /+}$ animals.

ObRflox/flox mice were viable, fertile, and indistinguishable from wild-type or $\mathrm{Ob} \mathrm{R}^{\mathrm{flox} /+}$ mice, indicating that the insertion of loxP sites into the introns flanking the first coding exon did not interfere with $\mathrm{ObR}$ function. To determine whether Cre-mediated deletion of the first coding exon results in a null allele, $O b R^{f l o x /+}$ mice were crossed with transgenic mice expressing Cre at an early embryonic stage (23). Mice with the genotype $\mathrm{ObR}^{4 /+}$, heterozygous for a germline deletion of the first coding exon (type I deletion), were generated and mated to produce homozygous $O b R^{\Delta / \Delta}$ mice, also referred to as ObR-null mice (Figure 1c). ObRnull mice are massively obese and indistinguishable from $d b^{3 J} / d b^{3 J}$ mice, which are also null for all ObR isoforms (Figure 1d) (28). These data confirm that the floxed allele is wild-type and that the deleted allele is null, thus validating the targeting strategy.

Neuron-specific ObR-knockout mice, designated $\mathrm{ObR}^{\mathrm{Syn} I} \mathrm{KO}$, were generated by two successive crosses (Figure 2a, and see Methods). These mice carry one floxed and one null allele so that Cre- mediated inactivation of only one allele, rather than two, is sufficient to delete $\mathrm{ObR}$ in a given cell. The $\mathrm{ObR}^{\mathrm{Syn} I} \mathrm{KO}$ mice were compared to littermate controls with the genotype $\mathrm{ObR}^{\Delta \text { flox }}$, SynI$\mathrm{Cre}(-)$, also referred to as heterozygotes. These mice were used as controls because some phenotypic changes have been observed in $d b /+$ mice (29). Data were also obtained from lean littermates with the genotypes $\mathrm{ObR}^{\mathrm{flox} /+}$, SynICre(+) or ObR ${ }^{\text {flox } /+}$, SynI-Cre(-), both of which are referred to as wild-type. Genotypes of all mice were determined using PCR and Southern blotting.

In the synapsinI-Cre transgenic line, expression of Cre recombinase is controlled by the rat synapsin I promoter. This promoter has been shown to drive Cre expression specifically in neurons (30). Crossing SynI-Cre(+) mice to LacZ indicator transgenic strains showed that Cre activity was first detectable at embryonic day (E) 12.5 and restricted to brain, spinal cord, and the dorsal root ganglion and was absent from astrocytes and glia (25). To confirm the tissue specificity of Cre-mediated recombination, a qualitative PCR assay was developed that was capable of detecting recombination between the lox sites flanking the first coding exon of ObR in genomic DNA. In ObR ${ }^{\text {flox } /+}$, synapsinI-Cre $(+)$ mice, Cre-mediated recombination was restricted to brain, hypothalamus, and spinal cord (Figure 2b). As previously indicated by reporter gene expression in SynI-CAT transgenic mice, low level recombination was also seen in testis (data not shown).

The weight of the $\mathrm{ObR}^{\mathrm{Syn}} \mathrm{KO}$ mice was compared to that of heterozygous mice at 4 months of age. Although the weights of ObR ${ }^{\text {SynI }} \mathrm{KO}$ mice, on average, were no different than heterozygotes, in both sexes a subset of the ObR ${ }^{\text {SynI }} \mathrm{KO}$ mice had an increased body weight (Figure 3a). The percent body fat of these mice (Figure 3a, yellow) was more than $2.5 \mathrm{SD}$ greater than the average percent body fat of heterozygotes (Figure 3a). This suggested the possibility that the extent of the knockout of ObR was variable and that the most obese $\mathrm{ObR}^{\text {Synn }} \mathrm{KO}$ animals had the lowest levels of ObR. To assess the efficiency of Cre-mediated recombination, real-time PCR assays (Taqman) were performed to quantitate ObR RNA using cDNA prepared from 
individual hypothalami (31). Previous data have suggested that in neurons, ObR is expressed at highest levels in hypothalamus and at much lower levels in other brain regions. This assay detected all forms of the leptin receptor. $\mathrm{ObR}^{\text {flox/+}}$, SynI-Cre(+) and $\mathrm{ObR}^{\text {flox/+, }}$ SynI-Cre(-) mice had equivalent levels of ObR expression, and thus both genotypes were designated as wild-type (Figure $3 b)$. As expected, ObR ${ }^{\Delta / \text { flox }}$, SynICre(-) mice, which have one allele inactivated in the germ line, have $50 \%$ as much RNA as wild-type mice $(P<0.006)$. The average level of ObR RNA was significantly lower in $\mathrm{ObR}^{\text {SynI }} \mathrm{KO}$ mice than in $\mathrm{ObR}^{\Delta \text { flox }}$, SynICre(-) heterozygote littermates (30\% vs. $50 \%$ of wild-type, respectively; $P=0.004$ ). Moreover, although many of the ObR ${ }^{\text {SynI }} \mathrm{KO}$ mice had levels of ObR RNA that were indistinguishable from heterozygotes, a subset of the $\mathrm{ObR}^{\mathrm{Syn} I} \mathrm{KO}$ mice had markedly reduced levels of ObR. These results confirmed that Cremediated recombination is variable, and indicated that in many animals there is no evident recombination.

We next considered whether the most

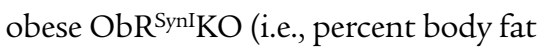
2.5 SD > heterozygotes) animals had lower levels of ObR RNA than the lighter ones. Analysis of the data indicated that percent body fat and body weight were significantly increased in each of six animals in which ObR RNA was less than $15 \%$ that of wild-type (Figure 3a, yellow) $(P<0.005$ for percent body fat; $P<0.05$ at all ages greater than 5 weeks for body weight). This confirmed that a significant deletion of ObR is associated with an obese phenotype. Furthermore, in those cases where the deletion is most extreme, a severely obese phenotype is evident. Two ObR ${ }^{\text {SynI } K O}$ females and one $\mathrm{ObR}^{\text {SynI }} \mathrm{KO}$ male had a greater than $97 \%$ reduction of ObR RNA, and each weighed more than $50 \mathrm{~g}$ at sacrifice, weights that approach, but do not equal, those of $d b / d b$ mice. In these studies, as well as studies of the $\mathrm{ObR}^{\mathrm{Alb}} \mathrm{KO}$ mice (see below), there were no lean mice with hypothalamic ObR RNA levels less than $15 \%$ that of wild-type, and there were no obese mice (obese defined as percent body fat > 2.5 SD above heterozygotes: $30.5 \%$ for males, $47.8 \%$ for females) with RNA levels greater than $15 \%$ that of wild-type. These data suggest that a near-normal body weight can be maintained until markedly reduced levels of ObR in hypothalamus are evident. In all following studies, the phenotype of the animals with less than $15 \%$ wild-type levels of hypothalamic ObR RNA was compared to heterozygotes. These mice are referred to as obese $\mathrm{ObR}^{\mathrm{Syn} I} \mathrm{KO}$ mice.

The weights of the obese $\mathrm{ObR}^{\mathrm{Syn}} \mathrm{KO}$ mice were significantly increased relative to heterozygotes at all time points greater than 5 weeks of age $(P<0.05)$.
At 5 months of age, males and females weighed $35 \%$ and $66 \%$ more than heterozygotes, respectively (Figure 4a). The increase in body weight was associated with increased adipose tissue mass and, as is also the case in $o b / o b$ and $d b / d b$ mice, decreased lean body mass (Table 1). At sacrifice, the percent body fat of ObR ${ }^{\text {SynI }} \mathrm{KO}$ mice was $39 \%$ in males and $60 \%$ in females, compared with $15 \%$ in male heterozygotes and $26 \%$ in female heterozygotes.

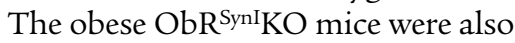
analyzed with respect to a number of other abnormalities associated with the $d b / d b$ mutation (Table 1). Plasma leptin concentrations were elevated 5.8-fold in obese ObR ${ }^{\text {SynI } K O}$ males $(P<0.05)$ and

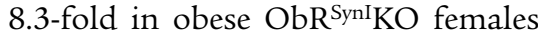
$(P<0.0001)$ and were highly correlated with percent body fat $(r=0.82, P<0.01$, $t$ test for correlation). In addition, plasma insulin was increased sixfold in

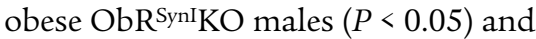

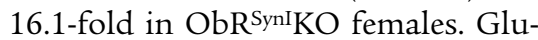
cose was increased 2.3-fold in

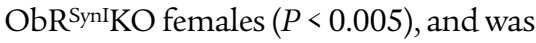
unchanged in $\mathrm{ObR}^{\mathrm{SynI}} \mathrm{KO}$ males. Plasma corticosterone was elevated 1.8-fold in $\mathrm{ObR}^{\text {SynI } K O}$ males and 2.4-fold in

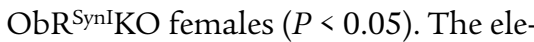
vation in plasma glucose and the greater elevation in corticosterone specific to females could be due to the females analyzed having a lower level of ObR RNA and being more obese. Of
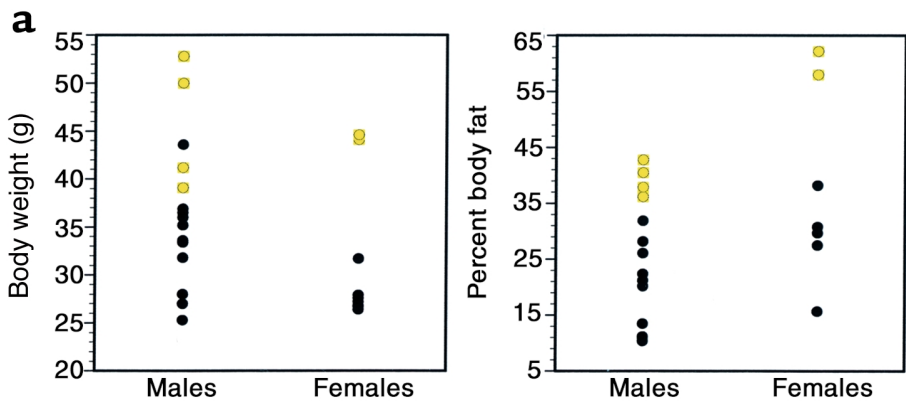

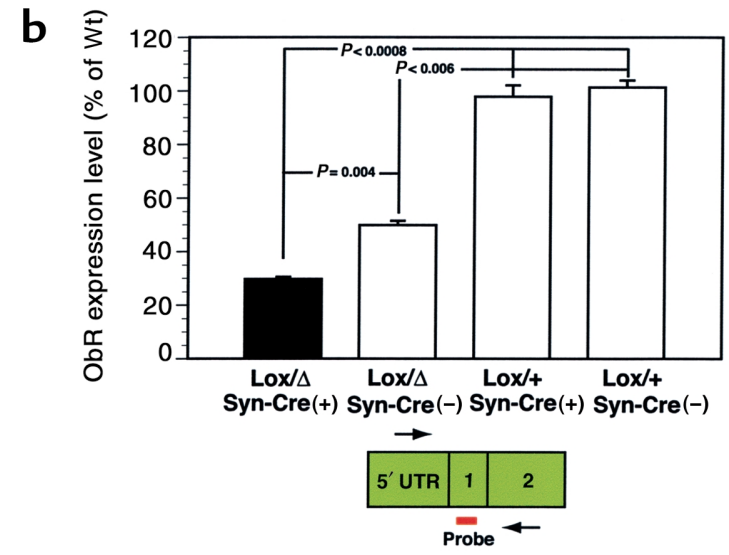

\section{Figure 3}

ObR RNA levels. (a) The distribution of body weight and percent body fat for male and female ObRSyn KO mice at 16 weeks of age. Those mice with less than $15 \%$ of ObR RNA (see b) are shown in yellow. (b) Expression levels were determined using Taqman real-time PCR with the ABI Prism 7700 Sequence Detection System. The locations of primers and fluorescent probe are as indicated. Primers were derived from sequences in the $5^{\prime}$ untranslated region and the second coding exon. The fluorescent probe is located within the first coding exon 1 . When this exon is deleted, no signal is generated. As a control for input amount, each cDNA sample was also amplified using primers and a probe for cyclophilin. Data were analyzed with ABI Sequence Detector software, and the levels of ObR were normalized to cyclophilin. Levels are represented as the percentage of the levels in $\mathrm{ObR}^{\text {flox } /+}$, Synl-Cre $(+)$ and $\mathrm{ObR}^{\mathrm{flox} /+}$, Synl-Cre(-) wild-type mice. Data represent the mean \pm SEM. At least 13 animals were analyzed for each genotype. $P$ values for comparisons between genotypes are indicated. 
note, an increased plasma corticosterone is unique to $o b / o b$ and $d b / d b$ mice, whereas other forms of rodent obesity are not generally associated with elevated corticosterone (32). Mutations in leptin or its receptor are also associated with infertility, and leptin has been shown to influence the hypothalamicpituitary-gonadal axis (33). Consistent with this, obese female ObR ${ }^{\text {SynI }} \mathrm{KO}$ mice had $43 \%$ reductions in plasma estradiol.

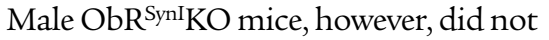
have reduced levels of plasma testosterone. This sexual dimorphism is consistent with the fact that leptin deficiency has a more profound effect on female reproduction than male reproduction (34). Finally, plasma thyroxine and triglycerides were not significantly different between mutant and control mice (data not shown).

Despite the marked obesity in this

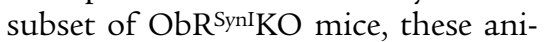
mals still weighed less than mice with germline deletions of ObR (Figure 4a; $P<0.05$ at all ages). These data suggest either that the knockout was incomplete even in the most obese animals or that leptin also acts at peripheral sites to reduce weight. To further assess the extent of the knockout in brain, levels of a number of hypothalamic neuropeptides were examined using Taqman assays (Figure 4b). Defective leptin signaling is associated with increased levels of hypothalamic AGRP, NPY, and $\mathrm{MCH}$
RNA and reduced levels of POMC and CART RNA (35-40). Comparisons of heterozygotes to obese $\mathrm{ObR}^{\mathrm{SynI}} \mathrm{KO}$ mice showed that AGRP and NPY levels were increased, whereas POMC and CART levels showed a trend toward being reduced. Although the reduction in POMC and CART RNA levels is not significant, the levels of these RNAs are only modestly decreased in $o b / o b$ and $d b / d b$ hypothalamus. $\mathrm{MCH}$ levels were indistinguishable between heterozygous

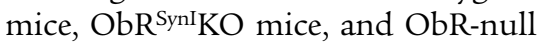
mice. The reported $80 \%$ increase in $\mathrm{MCH}$ RNA in $o b / o b$ mice is also more modest than the reported increases in AGRP and NPY RNA (38). Although the levels of AGRP and NPY were increased in the obese ObR ${ }^{\text {SynI }} \mathrm{KO}$ mice, they were less elevated than in null mice. This suggests that, despite the low levels of ObR RNA in these mice, some ObR-expressing neurons remained.

The data here indicate that leptin has direct effects on brain but leave open the possibility that leptin also has direct effects on peripheral tissues. To assess a possible role of ObR in liver, a peripheral tissue that has been suggested to be a direct target of leptin action, mice with a hepatocyte-specific knockout of ObR were generated $\left(\mathrm{ObR}^{\mathrm{Alb}} \mathrm{KO}\right)$. These mice were generated using the same breeding strategy as described for ObR ${ }^{\text {SynI } K O}$ mice (Figure $2 \mathrm{a}$ ). The albumin promoter was used to direct hepatocyte-specific expression of Cre recombinase (Figure 5a) (26). Genomic PCR performed on multiple tissues from $\mathrm{ObR}^{\text {flox/+ }}$ albuminCre(+) mice confirmed that Cre-mediated recombination was restricted to liver (Figure 5b). In contrast to $\mathrm{ObR}^{\text {SynI }} \mathrm{KO}$ mice, ObR ${ }^{\mathrm{Alb}} \mathrm{KO}$ mice did not manifest an increased weight even in those animals in which the levels of ObR RNA in liver (as measured using the aforementioned Taqman assay) were reduced to less than $30 \%$ of wild-type levels (9 of 12 males and 11 of 16 females analyzed) (Figure 5c). Because the liver is composed of a number of different cell types, and $80 \%$ of the mass is accounted for by hepatocytes, this likely represents a nearcomplete deletion in hepatocytes (41, 42). Inspection of the weight distributions showed that no $\mathrm{ObR}^{\mathrm{Alb}} \mathrm{KO}$ mice manifested an increased weight. Body composition was analyzed to determine whether hepatocyte-specific deletion of ObR had a subtle effect on body partitioning in $\mathrm{ObR}^{\mathrm{Alb}} \mathrm{KO}$ mice. Water mass, lean mass, and fat mass were all unchanged in these animals (Table 1). In both sexes, plasma leptin levels were within the normal range as were insulin, glucose, and corticosterone. Although $d b / d b$ and $O b R^{S y n I} \mathrm{KO}$ mice have enlarged fatty livers characteristic of leptin-deficient mice, the livers of $\mathrm{ObR}^{\mathrm{Alb}} \mathrm{KO}$ mice were grossly normal (Figure $5 \mathrm{~d}$ ). Wet liver weight and liver weight as a percentage of body weight were also
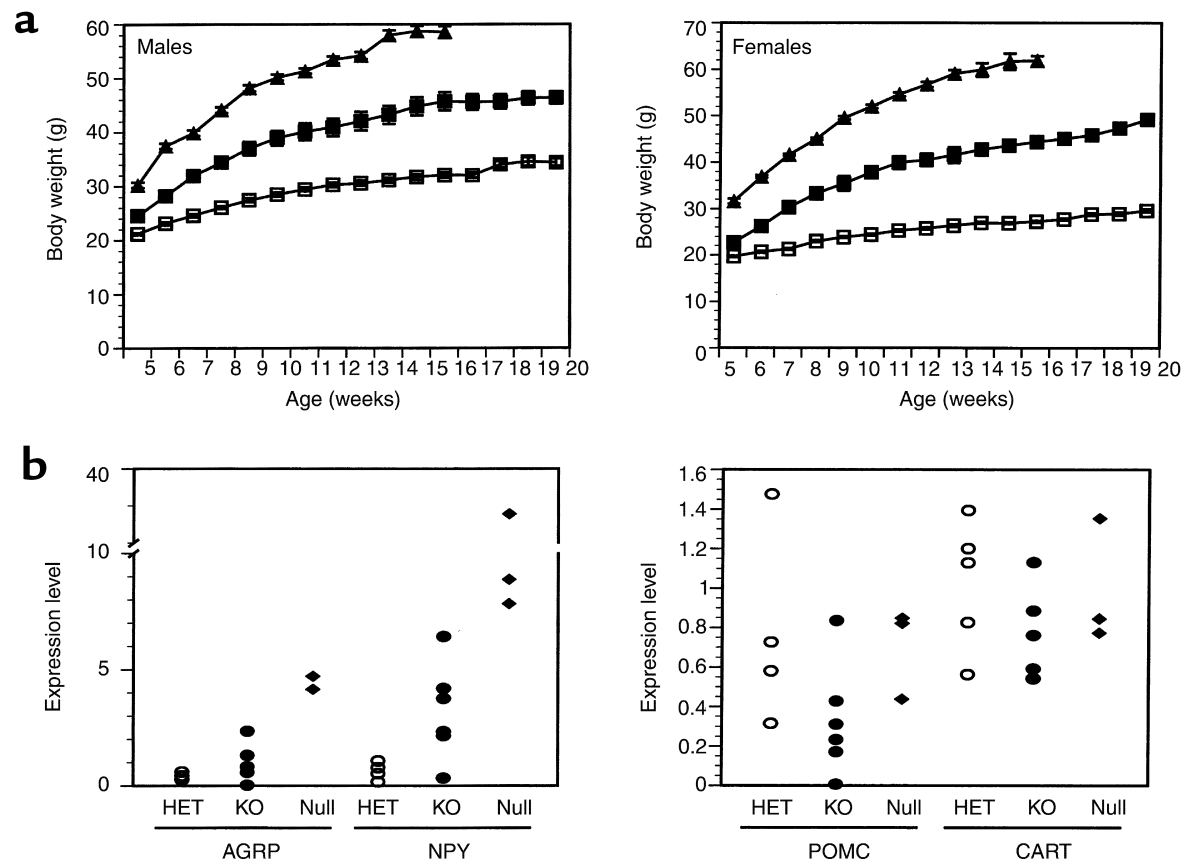

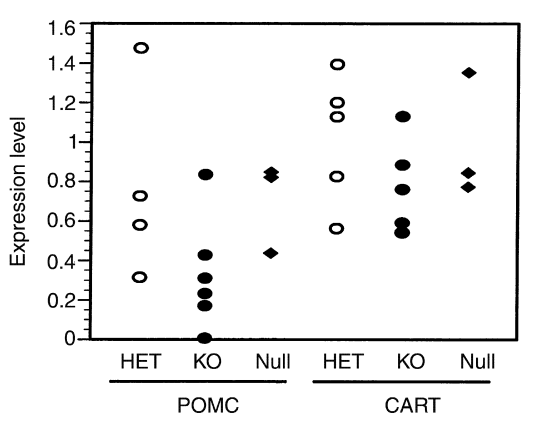

\section{Figure 4}

Obesity in a subset of ObR ${ }^{\text {Synl }} \mathrm{KO}$ mice. Significant obesity was evident in those ObR ${ }^{\text {Synl }} \mathrm{KO}$ mice that had $15 \%$ or less ObR RNA in the hypothalamus than did wildtype mice. (a) Weight curves of male and female ObR ${ }^{\text {Synl }} \mathrm{KO}$ mice (filled squares), heterozygote littermates (open squares), and ObR-null mice (filled triangles). Mice were weighed weekly from 5 weeks of age. Data represent the mean \pm SEM of four male and two female ObR ${ }^{\text {Synl }} \mathrm{KO}$ mice, seven male and nine female heterozygote littermates, and six male and five female ObR-null mice. For both sexes, ObR ${ }^{\text {Synl }} \mathrm{KO}$ versus heterozygotes, $P<0.05$ at all ages; $\mathrm{ObR}^{\text {Synl }} \mathrm{KO}$ versus ObR-null, $P<0.05$ at all ages. (b) Expression levels of AGRP, NPY, POMC, and CART were determined by Taqman and are expressed as normalized to cyclophilin. Levels were measured in $\mathrm{ObR}^{\text {Synl }} \mathrm{KO}$, heterozygotes (HET), and ObR-nulls. ( $n=5$ for heterozygotes, $n=6$ for knockouts, and $n=3$ for null mice.) 
a

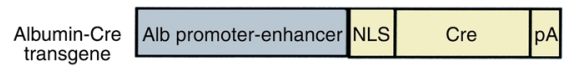

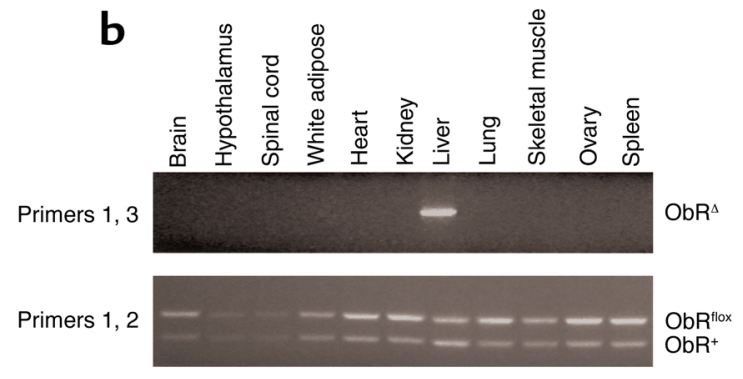

C

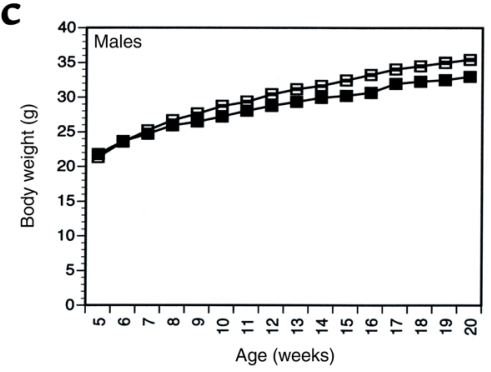

d

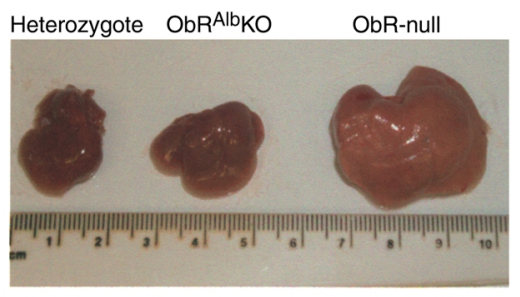

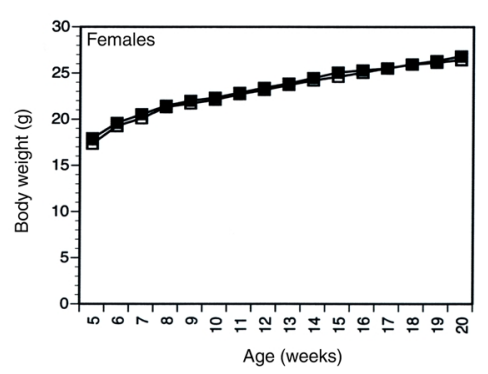

e

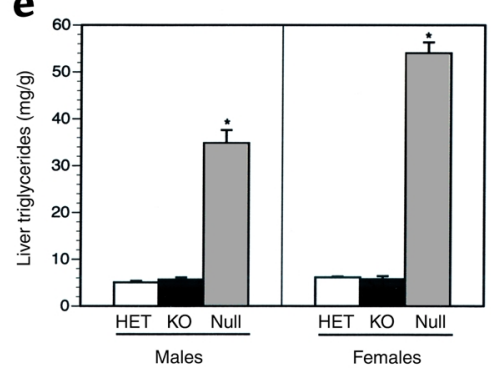

\section{Figure 5}

Normal body weight and liver phenotype in ObRAlbKO mice. (a) Schematic of Albumin-Cre transgene. (b) Genomic DNA was prepared from several tissues from $\mathrm{ObR}^{\text {flox } /+}$, Alb-Cre $(+)$ mice and was PCR-amplified using primers flanking the first coding exon of ObR as described in Figure $2 \mathrm{~b}$. (c) Weight curves of male and female ObR ${ }^{A l b} \mathrm{KO}$ mice (filled squares) and heterozygote littermates (open squares). Mice were

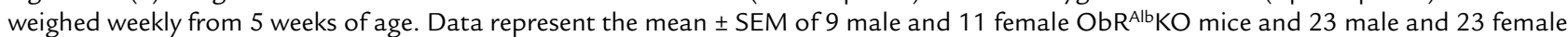
heterozygote littermates. For both sexes, ObR Alb KO versus heterozygotes, $P$ values were not significant at all ages. (d) Photographs of freshly dissected livers from representative mice with the given genotypes. (e) Liver triglycerides (milligrams of triglyceride per grams of liver) were determined for ObR AlbKO mice with less than 30\% of wild-type ObR RNA and heterozygote controls. Data represent the mean \pm SEM of six male and five female ObRAlbKO mice, five male and five female heterozygote controls, and six male and three female ObR-null mice. For both sexes, ${ }^{*} P<0.05$ for $\mathrm{ObR}^{\mathrm{Alb}} \mathrm{KO}$ and heterozygotes relative to ObR null.

unchanged between $\mathrm{ObR}^{\mathrm{Alb}} \mathrm{KO}$ mice and heterozygotes (data not shown). Quantitation of liver triglycerides showed no difference between $\mathrm{ObR}^{\mathrm{Alb}} \mathrm{KO}$ mice and heterozygote controls, whereas levels in ObR-null mice were significantly elevated relative to both of these groups $(P<0.05)$ (Figure $5 e)$. These data indicate that ObR expressed in hepatocytes is not likely to play a significant role in body weight homeostasis or the hepatic steatosis associated with the $d b$ mutation, and that the liver abnormalities observed in $o b / o b$ and $d b / d b$ mice are secondary to defective leptin signaling in the brain.

\section{Discussion}

In this study, two possible sites of leptin action were evaluated by analyzing the phenotype of mice with neuron and hepatocyte-specific knockouts of the leptin receptor. These data provide direct genetic evidence that leptin signals via direct effects on neurons. Separate data from studies of $d b^{K l s} / d b^{K l s}$ mice indicate that the specific absence of the ObRb form of the receptor results in a phenotype indistinguishable from leptin deficiency, demonstrating that $\mathrm{ObRb}$ is absolutely required for the weight-reducing effects of the hormone (4-6). ObRb is the only isoform that has all of the motifs necessary for signal transduction and is expressed at high levels in brain and a number of other tissues.

These findings suggest that leptin exerts its weight-reducing and some, or perhaps all, of its neuroendocrine effects via interactions with the $\mathrm{ObRb}$ form of the receptor in specific classes of neurons in brain. This is consistent with the high potency of leptin-administered ICV and the anatomic distribution of $\mathrm{ObRb}$ (see below) $(15,17)$. This conclusion is also consistent with data from experiments in which an NSE-ObRb transgene was able to partially complement the phenotype of $d b / d b$ mice (43). However, in this study, ObRb was overexpressed 30-fold in all neurons, with some leakiness in peripheral tissues.

The phenotype of the obese $O b R^{S y n} \mathrm{KO}$, while significant, did not reach that of ObR-null mice. In these animals, however, hypothalamic AGRP and NPY levels, while increased, were still not as high as in null mice, suggesting that there were still some residual 
Table 1

Body composition and neuroendocrine function in $\mathrm{ObR}^{\mathrm{Syn}} \mathrm{KO}$ and $\mathrm{ObR}^{\mathrm{Alb}} \mathrm{KO}$ mice

\begin{tabular}{|c|c|c|c|c|}
\hline & ObR Syn!KO & ObR ${ }^{\text {Synl Het }}$ & ObRAlbKO & ObRAlb Het \\
\hline \multicolumn{5}{|c|}{ Percent fat mass } \\
\hline Males & $39.4 \pm 0.7(4)$ & $15.4 \pm 0.8(8)^{\mathrm{B}}$ & $14.0 \pm 1.5(4)$ & $13.2 \pm 0.5(5)$ \\
\hline Females & $60.1 \pm 1.5(2)$ & $25.8 \pm 0.9(10)^{\mathrm{B}}$ & $15.4 \pm 0.9(7)$ & $15.6 \pm 1.1(5)$ \\
\hline \multicolumn{5}{|c|}{ Percent lean mass } \\
\hline Males & $19.7 \pm 0.6(4)$ & $25.7 \pm 0.2(8)^{\mathrm{B}}$ & $30.2 \pm 0.8(4)$ & $30.2 \pm 0.8(5)$ \\
\hline Females & $12.7 \pm 1.2(2)$ & $22.6 \pm 0.3(10)^{\mathrm{B}}$ & $28.0 \pm 0.7(4)$ & $27.5 \pm 0.7(5)$ \\
\hline \multicolumn{5}{|c|}{ Percent water mass } \\
\hline Males & $40.9 \pm 0.3(4)$ & $59.0 \pm 0.6(8)^{\mathrm{B}}$ & $55.8 \pm 1.3(4)$ & $56.6 \pm 0.6(5)$ \\
\hline Females & $27.2 \pm 0.3(2)$ & $51.6 \pm 0.6(10)^{\mathrm{B}}$ & $56.6 \pm 0.9(4)$ & $56.9 \pm 0.7(5)$ \\
\hline \multicolumn{5}{|c|}{ Leptin (ng/ml) } \\
\hline Males & $73.8 \pm 8.5(4)$ & $12.7 \pm 0.4(10)^{\mathrm{C}}$ & $13.1 \pm 0.8(9)$ & $13.9 \pm 0.7(15)$ \\
\hline Females & $125.0 \pm 0.7(2)$ & $15.1 \pm 0.8(12)^{\mathrm{B}}$ & $12.7 \pm 0.9(6)$ & $6.7 \pm 0.2(12)^{C}$ \\
\hline \multicolumn{5}{|c|}{ Insulin (ng/ml) } \\
\hline Males & $17.1 \pm 2.1(4)$ & $2.8 \pm 0.2(8)^{\mathrm{C}}$ & $1.9 \pm 0.1(9)$ & $1.6 \pm 0.1(6)$ \\
\hline Females & $40.1 \pm 6.5(2)$ & $2.5 \pm 0.2(8)$ & $0.8 \pm 0.1(4)$ & $0.7 \pm 0.1(5)$ \\
\hline \multicolumn{5}{|c|}{ Glucose (mg/dl) } \\
\hline Males & $158.3 \pm 9.3(4)$ & $165.3 \pm 4.9(10)$ & $80.3 \pm 2.3(7)$ & $89.1 \pm 2.5(8)$ \\
\hline Females & $282.6 \pm 32.3(2)$ & $123.1 \pm 4.7(8)^{\mathrm{B}}$ & $87.3 \pm 3.9(5)$ & $91.9 \pm 2.0(9)$ \\
\hline \multicolumn{5}{|c|}{ Corticosterone (ng/ml) } \\
\hline Males & $238.5 \pm 17.9(4)$ & $134.9 \pm 40.2(3)$ & $54.0 \pm 4.2(6)$ & $63.4 \pm 2.7(11)$ \\
\hline Females & $469.4 \pm 32.7(2)$ & $195.9 \pm 16.1(8)^{C}$ & $208.8 \pm 25.1(5)$ & $108.5 \pm 9.3(9)$ \\
\hline \multicolumn{5}{|c|}{ Estradiol (pg/ml) } \\
\hline Females & $3.9 \pm 0.3(2)$ & $6.8 \pm 0.8(3)$ & ND & ND \\
\hline
\end{tabular}

ObR-expressing cells. This leaves open the possibility that a truly complete brain-specific knockout (i.e., deletion of ObR on every neuron) could recapitulate the obese phenotype of ObR-null mice. The generation of such a knockout might require the simultaneous use of multiple different neuron-specific Cre-expressing lines, now under way.

In some studies, the expression of $\mathrm{ObRb}$ in brain was reported to be restricted to the hypothalamus, possibly implicating the hypothalamus as the principal target of leptin's weight-reducing effects. In hypothalamus, ObRb is enriched in the arcuate, ventromedial, dorsomedial, and paraventricular hypothalamic nuclei, all of which are known, based on studies of animals with stereotactic lesions, to play a role in regulating food intake and body weight (19, 44-46). However, in other studies, ObRb expression has also been reported in other brain sites including the brainstem, thalamus, and cerebellum. The possibility that one or more of these extrahypothalamic brain sites play important roles in the regulation of body weight can be tested using regionspecific or neuron-specific promoters to drive Cre expression in localized brain areas and specific cell types. In the hypothalamus, ObRb is expressed in functionally distinct classes of neurons, the best characterized being neurons that coexpress either NPY or $\alpha \mathrm{MSH}(47,48)$. Both of these neuropeptides exert potent effects on food intake and body weight. Experiments to test the effects of ObR deletion in these two types of neurons may allow an analysis of the functional importance of these two neuropeptides as downstream effectors of leptin signaling and could establish the extent to which these two cell types by themselves can fully account for the neural response to leptin.

A number of studies have suggested that leptin also has direct effects on liver $(20,21)$. The data presented here indicate that hepatocyte-specific deletion does not have any observable effects on body weight or body composition and does not result in any gross liver pathology. Furthermore ObR ${ }^{\mathrm{Alb}} \mathrm{KO}$ mice have equivalent amounts of liver triglycerides as do controls, indicating that the hepatic phenotype of $d b / d b$ mice is secondary to defective leptin signaling in brain. These findings do not exclude the possibility that hepatocyte ObR by itself serves functions, such as modulating leptin turnover.

Although these findings suggest that neurons are required for leptin's effects on body weight, they do not exclude the possibility that leptin also directly acts on other peripheral targets. In addition to regulating food intake, body weight, and the neuroendocrine axis, leptin can also modulate the immune system, bone metabolism, and angiogenesis (49-51). Indeed, ObRb is expressed in a number of peripheral tissues and direct effects of leptin on T cells, macrophages, pancreatic $\beta$-cells, and other cell types have been reported $(20,21,52)$. Other forms 
of ObR (ObRa, c, d, e) may play a role in transport of leptin across the blood brain barrier and choroid plexus, sites that express high levels of ObR. The function of ObR at these and other sites can now be assessed by crossing $O b R^{f l o x / f l o x}$ mice to transgenic lines that express Cre in a tissue-specific fashion. Such studies should enable an assessment of the relative role of direct effects of leptin on these tissues versus indirect effects via signaling in brain. In conclusion, these findings indicate that the brain is a direct target for the weight-reducing and neuroendocrine effects of leptin and that the liver pathology of $d b / d b$ mice is secondary to defective leptin signaling in neurons.

\section{Acknowledgments}

We thank $\mathrm{H}$. Westphal for adenovirus EIIA-Cre mice;J. Marth for synapsinI-Cre mice; $D$. Shmulewitz for assistance with data analysis; R. Sharma for assistance with liver photography; E. Asilmaz, S. Brown, M. Ishii, C. Li, S. Pinto, and A. Soukas for critical reading of this manuscript; and S. Korres for assistance in preparing this manuscript. P. Cohen was supported by NIH MSTP grant GM07739. J.M. Friedman was supported by NIH grant R01DK-41096.

1. Zhang, Y., et al. 1994. Positional cloning of the mouse obese gene and its human homologue. Nature. 372:425-432.

2. Friedman, J.M., and Halaas, J.L. 1998. Leptin and the regulation of body weight in mammals. Nature. 395:763-770.

3. Spiegelman, B.M., and Flier, J.S. 2001. Obesity and the regulation of energy balance. Cell. 104:531-543.

4. Tartaglia, L.A., et al. 1995. Identification and expression cloning of a leptin receptor, OB-R. Cell. 83:1263-1271.

5. Chen, H., et al. 1996. Evidence that the diabetes gene encodes the leptin receptor: identification of a mutation in the leptin receptor gene in $d b / d b$ mice. Cell. 84:491-495.

6. Lee, G.H., et al. 1996. Abnormal splicing of the leptin receptor in diabetic mice. Nature. 379:632-635.

7. Coleman, D.L. 1978. Obese and diabetes: two mutant genes causing diabetes-obesity syndromes in mice. Diabetologia. 14:141-148.

8. Coleman, D.L. 1982. Diabetes-obesity syndromes in mice. Diabetes. 31(Suppl. 1):1-6.

9. Yen, T.T., Allan, J.A., Yu, P-L., Acton, M.A., and Pearson, D.V. 1976. Triacylglycerol contents and in vivo lipogenesis of $o b / o b, d b / d b$, and $A^{v y} / a$ mice. Biochim. Biophys. Acta. 441:213-220.

10. Koteish, A., and Diehl, A.M. 2001. Animal models of steatosis. Semin. Liver Dis. 21:89-104.

11. Kaplan, M.L., and Leveille, G.A. 1981. Development of lipogenesis and insulin sensitivity in tissues of the $o b / o b$ mouse. Am. J. Physiology. 240:E101-E107.
12. Levin, N., Nelson, C., Gurney, A., Vandlen, R., and de Sauvage, F. 1996. Decreased food intake does not completely account for adiposity reduction after ob protein infusion. Proc. Natl. Acad. Sci. USA. 93:1726-1730.

13. Halaas, J.L., et al. 1995. Weight-reducing effects of the plasma protein encoded by the obese gene. Science. 269:543-546.

14. Pelleymounter, M.A., et al. 1995. Effects of the obese gene product on body weight regulation in ob/ob mice. Science. 269:540-543.

15. Campfield, L.A., Smith, F.J., Guisez, Y., Devos, R., and Burn, P. 1995. Recombinant mouse OB protein: Evidence for a peripheral signal linking adiposity and central neural networks. Science. 269:546-549.

16. Soukas, A., Cohen, P., Socci, N.D., and Friedman, J.M. 2000. Leptin-specific patterns of gene expression in white adipose tissue. Genes Dev. 14:963-980.

17. Halaas, J.L., et al. 1997. Physiological response to long-term peripheral and central leptin infusion in lean and obese mice. Proc. Natl. Acad. Sci. USA. 94:8878-8883

18. Ghilardi, N., et al. 1996. Defective STAT signaling by the leptin receptor in diabetic mice. Proc. Natl. Acad. Sci. USA. 93:6231-6235

19. Fei, H., et al. 1997. Anatomic localization of alternatively spliced leptin receptors (Ob-R) in mouse brain and other tissues. Prod. Natl. Acad. Sci. USA. 94:7001-7005.

20. Shimabukuro, M., et al. 1997. Direct antidiabetic effect of leptin through triglyceride depletion of tissues. Proc. Natl. Acad. Sci. USA. 94:4637-4641.

21. Kim, Y.B., Uotani, S., Pierroz, D.D., Flier, J.S., and Kahn, B.B. 2000. In vivo administration of leptin activates signal transduction directly in insulinsensitive tissues: overlapping but distinct pathways from insulin. Endocrinology. 141:2328-2339.

22. O'Gorman, S., Dagenais, N.A., Qian, M., and Marchuk, Y. 1997. Protamine-cre recombinase transgenes efficiently recombine target sequences in the male germ line of mice, but not in embryonic stem cells. Proc. Natl. Acad. Sci. USA. 94:14602-14607.

23. Lasko, M., et al. 1996. Efficient in vivo manipulation of mouse genomic sequences at the zygote stage. Proc. Natl. Acad. Sci. USA. 93:5860-5865.

24. DeFalco, J., et al. 2001. Virus-assisted mapping of neural inputs to a feeding center in the hypothalamus. Science. 291:2608-2613.

25. Zhu, Y., et al. 2001. Ablation of NF1 function in neurons induces abnormal development of cere bral cortex and reactive gliosis in the brain. Genes Dev. 15:859-876.

26. Pinkert, C.A., Ornitz, D.M., Brinster, R.L., and Palmiter, R.D. 1987. An albumin enhancer located $10 \mathrm{~kb}$ upstream functions along with its promoter to direct efficient, liver-specific expression in transgenic mice. Genes Dev. 1:268-276.

27. Gu, H., Marth, J.D., Orban, P.C., Mossmann, H., and Rajewsky, K. 1994. Deletion of a DNA polymerase $\beta$ gene segment in T cells using cell typespecific gene targeting. Science. 265:103-106.

28. Lee, G-H., et al. 1997. Leptin receptor mutations in $129 d b^{3 J} / d b^{3 J}$ mice and NIH $f a^{c p} / f a^{c p}$ rats. Mamm. Genome 8:445-447.

29. Coleman, D.L. 1979. Obesity genes: beneficial effects in heterozygous mice. Science. 203:663-665.

30. Hoesche, C., Sauerwald, A., Veh, R.W., Krippl, B., and Kilimann, M.W. 1993. The 5 '-flanking region of the rat synapsin I gene directs neuron-specific and developmentally regulated reporter gene expression. J. Biol. Chem. 268:26494-26502.

31. Livak, K.J., Flood, S.J., Marmaro, J., Giusti, W., and Deetz, K. 1995. Oligonucleotides with fluorescent dyes at opposite ends provide a quenched probe system for detecting PCR products and nucleic acid hybridization. PCR Methods Appl. 4:357-362.
32. Bray, G.A., and York, D.A. 1979. Integration of energy intake and expenditure in animals and man: the autonomic and adrenal hypothesis. Physiol. Rev. 59:719-809.

33. Chehab, F.F., Mounzih, K., Lu, R., and Lim, M.E. 1997. Early onset of reproductive function in normal female mice treated with leptin. Science. 275:88-90.

34. Ewart-Toland, A., Mounzih, K., Qiu, J., and Chehab, F.F. 1999. Effect of the genetic background on the reproduction of leptin-deficient obese mice. Endocrinology. 140:732-738.

35. Ollmann, M.M., et al. 1997. Antagonism of central melanocortin receptors in vitro and in vivo by agouti-related protein. Science. 278:135-138.

36. Shutter, J.R., et al. 1997. Hypothalmic expression of ART, a novel gene related to agouti, is up-regulated in obese and diabetic mutant mice. Genes Dev. 11:593-602.

37. Stephens, T.W., et al. 1995. The role of neuropeptide $\mathrm{Y}$ in the antiobesity action of the obese gene product. Nature. 377:530-534.

38. Qu, D., et al. 1996. A role for melanin-concentrating hormone in the central regulation of feeding behavior. Nature. 380:243-247.

39. Thornton, J.E., Cheung, C.C., Clifton, D.K., and Steiner, R.A. 1997. Regulation of hypothalamic proopiomelanocortin mRNA by leptin in $o b / o b$ mice. Endocrinology. 138:5063-5066.

40. Kristensen, P., et al. 1998. Hypothalamic CART is a new anorectic peptide regulated by leptin. Nature. 393:72-76.

41. Gumucio, J.J., Berkowitz, C.M., Webster, S.T., and Thornton, A.J. 1996. Structural and functional organization of the liver. In Liver and biliary disease N. Kaplowitz, editor. Williams and Wilkins. Baltimore, Maryland, USA. 3-19.

42. Weibel, E.R., Staubli, W., Gangi, H.R., and Hess, F.A. 1969. Correlated morphometric and biochemical studies on the liver cell. I. Morphometric model, stereologic methods, and norma morphometric data for rat liver. J. Cell. Biol. 42:68-91.

43. Kowalski, T.J., Liu, S-M., Leibel, R.L., and Chua, S.C. 2001. Transgenic complementation of leptin receptor deficiency. Diabetes. 50:425-435.

44. Elmquist, J.K., Ahima, R.S., Maratos-Flier, E., Flier, J.S., and Saper, C.B. 1997. Leptin activates neurons in ventrobasal hypothalamus and brainstem. Endocrinology. 138:839-842.

45. Elmquist, J.K., Bjorbaek, C., Ahima, R.S., Flier, J.S., and Saper, C.B. 1998. Distributions of the leptin receptor mRNA isoforms in the rat brain. J. Comp. Neurol. 395:535-547.

46. Hetherington, A.W., and Ranson, S.W. 1942. The spontaneous activity and food intake of rats with hypothalamic lesions. Am. J. Physiol. 136:609-617.

47. Mercer, J.G., et al. 1996. Coexpression of leptin receptor and preproneuropeptide $\mathrm{Y}$ mRNA in arcuate nucleus of mouse hypothalamus. J. Neuroendocrinology. 8:733-735.

48. Cheung, C.C., Clifton, D.K., and Steiner, R.A. 1997. Proopiomelanocortin neurons are direct targets for leptin in the hypothalamus. Endocrinology. 138:4489-4492.

49. Lord, G.M., Matarese, G., Howard, J.K., Bloom S.R., and Lechler, R.I. 1998. Leptin modulates the $\mathrm{T}$-cell immune response and reverses starvation induced immunosuppression. Nature. 394:897-891.

50. Ducy, P., et al. 2000. Leptin inhibits bone formation through a hypothalamic relay: a central control of bone mass. Cell. 100:197-207.

51. Sierra-Honigmann, M., et al. 1998. Biologic action of leptin as an angiogenic factor. Science. 281:1683-1686.

52. O'Rourke, L., Yeaman, S.J., and Shepherd, P.R. 2001. Insulin and leptin acutely regulate cholesterol ester metabolism in macrophages by novel signaling pathways. Diabetes. 50:955-961. 